\title{
Langzeit-Therapie mit Johanniskraut: Wirksamkeit und Rezidivprophylaxe doppelblind nachgewiesen
}

In einer doppelblinden, placebokontrollierten Multicenterstudie wurden Wirksamkeit und Sicherheit des Hypericumextrakts WS ${ }^{\circledR} 5570$ zur Rezidivprophylaxe während einer insgesamt 18-monatigen Therapie bei Patienten mit wiederholt rezidivierenden Depressionen geprüft. In die Studie aufgenommen wurden erwachsene Patienten mit rezidivierender Form einer moderaten "major depression", einem HAMD-Gesamtscore von $\geq 20$ und $\geq 3$ vorausgehenden depressiven Episoden während 5 Jahren. Nach einer 6-wöchigen einfachverblindeten Therapie mit WS ${ }^{\circledR} 5570$ (Hyperiplant ${ }^{\circledR}, 3$ x 300 mg/Tag) wurden die Patienten mit einem Wert $\leq 2$ bei “Verbesserung" auf der Clinical Global Impressions (CGI)-Skala und einer Abnahme beim HAMD-Gesamtscore $\leq 50 \%$ weitere 26 Wochen auf WS ${ }^{\circledR} 5570$ (3 x 300 mg/Tag) oder Placebo randomisiert. Die Daten von 426 Patienten wurden ausgewertet. Rückfallquoten während der Dauertherapie lagen bei $18,1 \%$ unter WS ${ }^{\circledR} 5570$ und bei 25,7\% unter Placebo. Die Durchschnittszeit bis zum Rezidiv lag bei $177 \pm 2,8$ Tagen für WS ${ }^{\circledR} 5570$ und $163 \pm 4,4$ Tagen für Placebo $(p=0,034)$. Patienten unter WS ${ }^{\circledR} 5570$ zeigten im Vergleich zu Placebo günstigere Werte nach HAMD und CGI. Während der Langzeit-Erhaltungstherapie wurde eine ausgeprägte prophylaktische Wirkung von WS ${ }^{\circledR} 5570$ bei Patienten mit einem frühen Beginn bzw. mit hoher Chronizität der Depression beobachtet. Die Anzahl unerwünschter Wirkungen unter WS ${ }^{\circledR} 5570$ war der unter Placebo vergleichbar.

Major Depression ist eine weit verbreitete und oftmals chronische Erkrankung; die Rezidivrate liegt bei mehr als $50 \%$. Prävalenz und Schweregrad krankheitsbedingter Morbidität steigen mit der Anzahl der Rezidive an, während der Zeitraum zwischen zwei aufeinanderfolgenden Episoden und die Ansprechempfindlichkeit auf eine Antidepressionstherapie gleichzeitig abnehmen: Hat der Patient bereits 3 Episoden erlitten, ist die Wahrscheinlichkeit einer weiteren Episode innerhalb der nächsten zwei Jahre grösser als 95\%. Trotz offensichtlich negativer Auswirkungen auf Sozialkontakte und Lebensqualität ist die Depression nach wie vor untertherapiert: Die erste in der EU durchgeführte paneuropäische Studie zeigte, dass lediglich $25 \%$ der therapiebedürftigen Patienten Antidepressiva erhalten, und eine kürzlich in England durchgeführte Kohortenstudie mit einem Follow-up von 11 Jahren zeigt, dass sich das Langzeitergebnis bei Depression in den letzten 20 Jahren nicht signifikant verändert hat. Der Grund für eine unzureichende Therapie kann eine nicht korrekte Diagnose oder ein nicht ausreichender Behandlungszeitraum sein.

Während es eine Fülle von Studien zur Wirksamkeit zahlreicher Antidepressiva in der Kurzzeittherapie gibt, besteht ein Mangel an methodologisch adäquaten Studien zur Wirkung dieser Medikamente während einer Langzeitprophylaxe. Eine Re-Randomisierung von Respondern nach Akuttherapie ist dabei zum Beweis einer Langzeitwirksamkeit unbedingt erforderlich.

Die antidepressive Wirkung von Hypericumextrakt in der Akuttherapie einer milden bis moderaten Depression ist gut belegt. In den meisten Reviews wurden Unterschiede bei den Effektgrössen festgestellt. Diese lassen sich zumindest zum Teil auf den Einsatz unterschiedlicher Extrakte mit unterschiedlichem Hypericin- und Hyperforingehalt zurückführen, dem die antidepressive Wirkung hauptsächlich zugeschrieben wird: Somit sind die einzelnen Extrakte hinsichtlich ihrer Wirksamkeit nicht austauschbar. Trotz einer beachtlichen Popularität des Phytotherapeutikums in der klinischen Praxis wurde bisher keine Studie publiziert, in der die Wirkung einer Langzeit-Prophylaxe placebokontrolliert und doppelblind abgesichert wurde.

\section{Studienteilnehmer}

62 Psychiatrie- und Allgemeinpraxen in Deutschland und Schweden nahmen an der Studie teil. Studienteilnehmer (Männer und Frauen) mussten zwischen 18 und 65 Jahre alt, an einem Rezidiv einer major depression erkrankt sein und ihre Krankengeschichte musste 2 oder 3 frühere Episoden nach ICD-10- und DSM-IV-Kriterien aufweisen. Ein HAMD-Gesamtscore von $\geq 20$ Punkten musste gegeben sein.

\section{Studienprotokoll, Studiendesign und Studienziele}

Geeignete Patienten wurden in eine 1-wöchige einfachblinde Placebo-Washout-Phase aufgenommen. Patienten, die danach immer noch die Diagnosekriterien für die Aufnahme, insbesondere den erforderlichen HAMD-Score, erfüllten, erhielten eine einfachblinde 6-wöchige Akuttherapie mit $3 \times 300$ mg/Tag WS ${ }^{\circledR} 5570$ (Hyperiplant ${ }^{\circledR}$ ). Im Anschluss wurden die Therapieresponder im Verhältnis 2:1 auf eine 26wöchige doppelblinde Weiterbehandlung mit $3 \times 300 \mathrm{mg} / \mathrm{Tag}$ WS ${ }^{\circledR} 5570$ oder Placebo randomisiert. Das Ansprechen der Therapie war definiert als eine starke/sehr starke Verbesserung beim CGI-Score sowie eine Verringerung des HAMD-Gesamtscore um mindestens 50\% im Vergleich zum Therapiebeginn. Nach Abschluss dieser Behandlung wurden die Patienten ohne Rezidive einer 52-wöchigen doppelblinden Erhaltungstherapie zugeführt, d.h. re-randomisiert im Verhältnis 1:1 auf eine Erhaltungstherapie mit 3x300 mg/ Tag WS ${ }^{\circledR} 5570$ oder Placebo.

\section{Ergebnisse}

Bei jeder Patientenvisite wurde der Schweregrad der Depression mittels HAMD, BDI (Beck Depression Inventory) und CGI beurteilt. Primärer Endpunkt während der Weiterbehandlung war der Zeitraum bis zum Rezidiv. Sekundäre Endpunkte umfassten den Zeitraum bis zum Rezidiv während der Erhaltungstherapie sowie die Rezidivraten und HAMD-, BDI- und CGI-Werte bei der jeweiligen letzten Visite während der Weiterbehandlung und Erhaltungstherapie. Die Verträglichkeit der Medikation wurde bei jeder Visite durch Befragung der Patienten nach unerwünschten Ereignissen ermittelt. 


\section{Wirksamkeit}

In der Gesamtauswertung der Weiterbehandlung zeigt sich, dass es unter Hypericumextakt (im Vergleich zu Placebo) länger dauert bis zu einem erneuten Rezidiv und dass die Rezidivrate im Vergleich zu Placebo niedriger ist $(\mathrm{p}=0.021$ zugunsten von Hypericumextrakt) (Abb. 1).

Während der einfachblinden Akuttherapie mit $\mathrm{WS}^{\circledR}$ 5570 zeigten die Patienten, die später auf den Pflanzenextrakt randomisiert wurden, und diejenigen, die später auf Placebo randomisiert wurden, vergleichbare Verbesserungen im HAMD-Gesamtscore um jeweils 15 Punkte. Während der doppelblinden Weiterbehandlung zeigten die Patienten unter WS $^{\circledR} 5570$ eine weitere Verbesserung im HAMD-Gesamtscore, während die Werte in der Placebogruppe fast unverändert blieben ( $\mathrm{p}=0.04)$ (Abb. 2).

16 von 93 Patienten in der $\mathrm{WS}^{\circledR}$ 5570-Gruppe $(17,2 \%)$ und 20 von 98 Patienten in der Placebogruppe $(20,4 \%)$ erlitten ein Rezidiv im Verlauf der Langzeitprophylaxe. Für Hypericumextrakt wurden besonders niedrige Rezidivraten bei Patienten mit einem frühen Beginn der depressiven Episode beobachtet, d.h. bei denjenigen mit einer längeren Krankheitsdauer (Alter beim Entritt der ersten Episode $\leq 42$ Jahre: WS ${ }^{\circledR} 5570$ Rezidiv 11,1\%, Placebo 29,4\%, p=0,01 einseitig).

Unerwünschte Ereignisse traten in beiden Gruppen mit vergleichbarer Häufigkeit auf.

\section{Diskussion}

Die vorliegende Studie zeigt die prophylaktische Wirksamkeit von Hypericumextrakt WS ${ }^{\circledR} 5570$ bei Patienten, die nach Genesung von einer akuten Episode einer major depression mit dem Extrakt weiterbehandelt wurden. Während einer solchen sechsmonatigen Weiterbehandlung bewirkte eine Tagesdosis von $900 \mathrm{mg} \mathrm{WS}^{\circledR} 5570$ ein um $30 \%$ geringeres Rezidivrisiko im Vergleich zu Placebo und zeigte eine positive Wirkung auf sämtliche untersuchten psychiatrischen Bewertungsskalen.

Subgruppenanalysen zeigten, dass die Rezidivprophylaxe mit WS ${ }^{\circledR} 5570$ bei Patienten mit einem anfangs stark ausgeprägten Krankheitsbild ebenso wirksam war wie bei weniger schwer erkrankten Patienten. In der Placebogruppe hingegen hatten die Patienten mit einem anfangs stark ausgeprägten Krankheitsbild ein um etwa 50\% höheres Rezidivrisiko während der Weiterbehandlung. Studienteilnehmer mit einem anfänglichen HAMD-Gesamtscore $\geq 25$ Punkte hatten ein um 38\% niedrigeres Rezidiv-Risiko und die Studienteilnehmer, bei denen der HAMD-Gesamtscore nach Abschluss der initialen Akuttherapie immer noch > 7 Punkte betrug, ein um 31\% geringeres Rezidiv-Risiko, wenn sie mit Hypericumextrakt weiterbehandelt wurden.

Dieses Ergebnis stimmt mit der klinischen Erfahrung überein, derzufolge die besten Prophylaxeresultate dann zu erwarten sind, wenn das erfolgreich während der Akuttherapie eingesetzte Präparat weiter in der vollen Therapiedosis während einer Langzeitprophylaxe verabreicht wird, sofern vom Patienten gut vertragen.

Die weitverbreitete Unterlassung einer Rezidivprophylaxe mit Antidepressiva scheitert möglicherweise häufig an

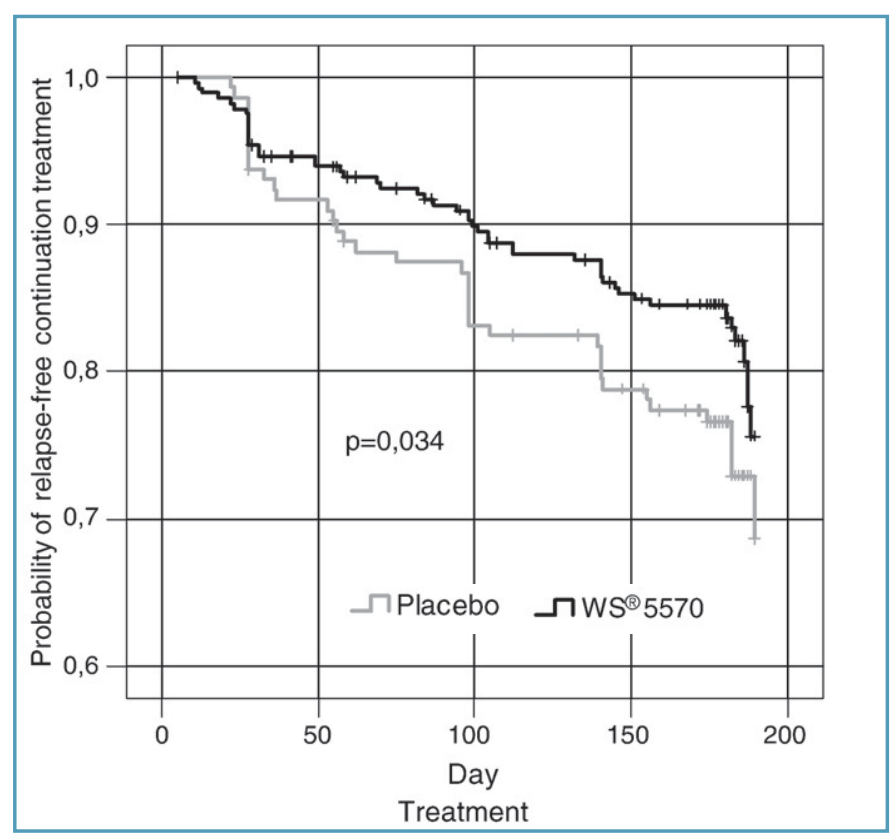

Abb. 1. Weiterbehandlung: Zeit bis zum Auftreten eines neuen Rezidivs.

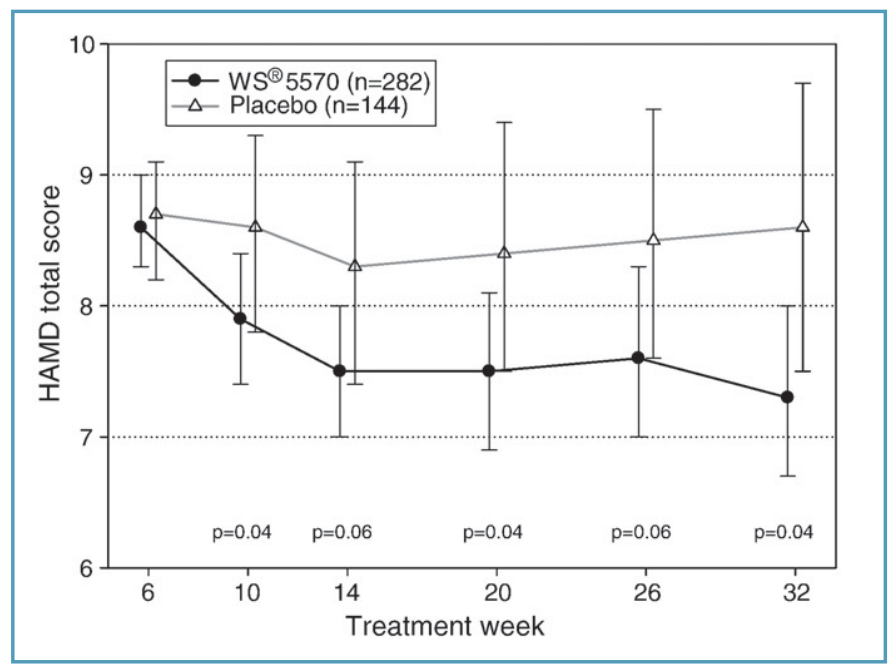

Abb. 2. Weiterbehandlung: Entwicklung des HAMD-Score.

der Angst vor Nebenwirkungen, die den Patienten belasten. Die vorliegende Studie zeigt eindrucksvoll, dass keine spezifischen Nebenwirkungen zu erwarten sind, wenn $\mathrm{WS}^{\circledR}$ 5570 in einer therapeutischen Dosis zur Langzeit-Prophylaxe eingesetzt wird. Bei insgesamt 703 Patienten unter Hypericumextrakt WS ${ }^{\circledR} 5570$ traten keine unerwarteten arzneimittelspezifischen Risiken oder Intoleranzprobleme auf.

Kasper S, Volz HP, Möller HJ, Dienel A, Kieser M: Continuation and longterm maintenance treatment with Hypericum extract WS ${ }^{\circledR} 5570$ after recovery from an acute episode of moderate depression - A doubleblind, randomized, placebo controlled long-term trial. European Neuropsychopharmacology 2008;18:803-813. 